\title{
CONDIÇÕES TÉCNICO-ECONÔMICAS PARA VIABILIZAR O EMPREGO DE AÇOS PLANOS DE ELEVADA RESISTÊNCIA MECÂNICA EM APLICAÇÕES PRÁTICAS
}

Willy Ank de Morais '

Herbert Christian Borges ${ }^{2}$

\section{Resumo}

Componentes veiculares e partes produzidas com materiais de alta resistência levam a menor peso, menor consumo de combustível e melhor desempenho desses veículos, pela possibilidade de diminuição da espessura desses componentes. Nesta linha, existem muitos desenvolvimentos que buscam a substituição de materiais de baixa resistência por materiais de alta resistência, para melhorar o desempenho dos veículos. As novas ligas, baseadas no alumínio e no titânio e os desenvolvimentos de novos aços microligados (ARBL) e/ou de aços com uma fase transformada ou adicional (Duplex, Dual-phase, TRIP e TWIP), têm este objetivo básico. Porém, existem considerações importantes que devem ser feitas para tornar essa substituição possível: questões associadas ao processo de fabricação, uso prático final desses equipamentos e componentes, e a viabilidade econômica. Este trabalho apresenta as principais considerações que devem ser feitas para fazer substituições realísticas em componentes de uso rodoviário e apresenta exemplos reais com aços. Nos casos apresentados, as substituições ocorreram com sucesso, não só pela diminuição do peso da estrutura, mas também pelo o aumento da resistência mecânica total da estrutura, com mínimo impacto na produção e ainda com redução do custo final do produto.

Palavras-chave: Aços; Aços microligados; Materiais; Veículos.

\section{TECNHICAL AND ECONOMIC CONDITIONS TO MAKE FEASIBLE THE USE OF HIGH STRENGTH STEELS IN PRATICAL APPLICATIONS}

\begin{abstract}
Vehicular equipments and parts made with high strength materials can provide low weight, fuel economy and better performance to vehicles. The main key is thickness reduction obtained as consequence of improved steel strength used in these components. In that way, there are several developments aiming materials replacement to improve vehicular performance. New alloys, based on aluminum and titanium, and developments on new microalloyed steels (HSLA) and transformed/additional phase steels (Duplex, Dual-phase, TRIP e TWIP), have been used for improve vehicular performance. However, there are important considerations need to be done to make this replacement possible: manufacture issues, practical final use of these equipments and parts, and economical viability of course. This work presents the main considerations to make practical materials replacement of truck components and case studies exemplify the ideas presented here. In the replacements cases showed, success was obtained not only by weight savings, but additionally by increase of final structure resistance, with minimum impact on manufacturing and besides, with lowering final cost of product.
\end{abstract}

Key words: Steels; Microalloyed steels; Materials; Vehicles.

\section{INTRODUÇÃO}

Muitos dos trabalhos de redução de peso dos veículos partem do simples conceito de que, substituindo um material de menor resistência por outro de maior resistência, é possível utilizar materiais mais finos e leves e que apresentam o mesmo grau de resistência absoluta, mas que levam a uma redução do peso total do veículo. Este tipo de análise tem sido utilizado na disputa entre diferentes materiais estruturais e foi um dos principais pilares para $\circ$ desenvolvimento do projeto ULSAB. ${ }^{(1)}$

\footnotetext{
'Doutorando, Mestre em Eng. ${ }^{a}$ Metalúrgica e de Materiais, Eng. Metalurgista, Téc. em Metalurgia, Analista da Usiminas, Professor da Faculdade de Engenharia da UNISANTA e Vice-Diretor da Divisão Técnica "Aplicações de Materiais" da ABM. Rua Onze de Junho, I3I Apto I54. Itararé, Cep II320-I60, São Vicente, SP, Brasil.E-mail: willyank@unisanta.br; willy.morais@usiminas.com.

${ }^{2}$ Mestrando em Eng. ${ }^{a}$ Metalúrgica, Eng. Metalurgista, Analista de Assistência Técnica da Usiminas. Av. Senador Vitorino Freire, 709. Jd. Rei,

Cep 09910-550, Diadema, SP.E-mail: herbert.borges@usiminas.com
} 
A correta seleção do material pode ter grande impacto no peso dos veículos, sendo considerável o peso total de metal utilizados neles. Em um trabalho encomendado pelo IBS e realizado pelo IPT em 2005, (2) utilizaram-se dois veículos de passeio de fabricação nacional e verificou-se que os peso total dos componentes em aço nestes veículos foi de $503 \mathrm{~kg}$ e $594 \mathrm{~kg}$, o que representa uma média de $53 \%$ em um peso total de $903 \mathrm{~kg}$ e $1.180 \mathrm{~kg}$, respectivamente..$^{(2)}$ Outros trabalhos descrevem a participação do aço nas diversas partes dos automóveis. Em um destes exemplos ${ }^{(3)} \mathrm{O}$ aço utilizado na carroceria possui uma participação em torno de $25 \%$ do peso total de um carro de passeio médio, seguido por $18 \%$ para o motor e transmissão e por $12 \%$ para o sistema de suspensão, resultado similar ao total médio de $53 \%$ obtido no trabalho do IBS. (2)

Quando se analisam os números citados nesses trabalhos e considera-se a distribuição de peso nas diversas partes do automóvel citado como válida nos automóveis nacionais, ${ }^{(2,3)}$ pode-se fazer uma estimativa do potencial de ganho só pela utilização de um aço $27 \%$ mais resistente na carroceria (Tabela I).

Esse ganho em peso poderia ser obtido, por exemplo, ao se substituir um aço DIN EN 10268 H240LA, ${ }^{(4)}$ com limite de escoamento (LE) mínimo de $240 \mathrm{MPa}$, por outro da mesma qualidade, porém do grau H320LA, com LE mín. de $320 \mathrm{MPa}$ (incremento de $33 \%$ ). Deve-se salientar, neste exemplo, que nem todo ganho de resistência foi explorado, o que dota esse automóvel de um incremento líquido em resistência que aumenta sua longevidade, especialmente sob condições de fadiga. ${ }^{(5)}$

Entretanto, somente a substituição de um material de menor resistência mecânica por outro de maior resistência mecânica, não garante o sucesso na aplicação. Existem outras considerações que devem ser feitas para que a substituição tenha sucesso e que a vantagem do ganho em peso não agregue dificuldades em outros aspectos na fabricação e no uso final do veículo. Considerações principalmente a respeito do custo, mas também da conformabilidade, ${ }^{(6)}$ soldabilidade e rigidez da estrutura devem ser feitas para implementar substituições práticas que levem a resultados concretos, como exemplificado neste trabalho.

\section{DESENVOLVIMENTO}

\section{I Particularidades dos Aços Planos}

Além das características químicas e mecânicas, existem outras características aplicáveis aos aços planos, que não são diretamente explicitadas na norma pela qual o aço é especificado, como nas normas DIN ENI0268,(4) ASTM A36(7) e ASTM A572. ${ }^{(8)}$ Questões relacionadas à tolerância dimensional, planicidade e de forma são especificadas em outras normas complementares às normas dos aços, como na norma ASTM A568,(9) que geralmente fazem vínculo à classificação do grau de resistência do aço, como ilustrado na Tabela 2. Assim, a alteração do grau de resistência do aço pode exigir que se alterem as tolerâncias de fornecimento do material.

Por outro lado, para otimizar o processo produtivo é muito utilizada a chamada "espessura padrão", que é um pequeno prêmio que os produtores e revendedores de metais planos oferecem para aqueles que adquirem material em espessuras mais costumeiras à produção e ao mercado. No caso dos aços planos, alguns exemplos das chamadas "espessuras padrão" são: $2,65 \mathrm{~mm}$; $3,00 \mathrm{~mm} ; 4,75 \mathrm{~mm} ; 5,00 \mathrm{~mm} ; 6,30 \mathrm{~mm} ; 8,00 \mathrm{~mm}$ e $9,50 \mathrm{~mm}$.

$\mathrm{Na}$ eventual redução de espessura, após a seleção de um material de maior resistência mecânica, é aconselhável que a espessura final pertença ao conjunto das "espessuras padrão". Isso limita as possibilidades de escolhas de espessura, mas permite $\circ$ uso de um material mais facilmente obtenível no mercado, favorecendo um maior potencial de diminuição de custo.

Tabela I. Potencial diminuição de peso de um automóvel de passeio ${ }^{(2,3)}$ pela redução da espessura do aço utilizado na carroceria

\begin{tabular}{|c|c|c|c|c|c|c|c|c|}
\hline \multirow[t]{3}{*}{ Automóvel } & \multirow{3}{*}{$\begin{array}{c}\begin{array}{c}\text { Peso do } \\
\text { automóvel }\end{array} \\
(\mathrm{kg})\end{array}$} & \multicolumn{4}{|c|}{ Participação do aço } & \multirow{2}{*}{\multicolumn{3}{|c|}{$\begin{array}{l}\text { Redução de espessura } \\
\text { (peso) na carroceria }\end{array}$}} \\
\hline & & \multicolumn{2}{|c|}{ no automóvel } & \multicolumn{2}{|c|}{ na carroceria } & & & \\
\hline & & $(\%)$ & (kg) & (\%) & (kg) & (\%) & (kg) & (\% total) \\
\hline A & 903 & $55,7 \%$ & 503 & $25 \%$ & 226 & $27 \%$ & 61 & $6,8 \%$ \\
\hline B & 1.180 & $50,3 \%$ & 594 & $25 \%$ & 295 & $27 \%$ & 80 & $6,8 \%$ \\
\hline Média & 1.042 & $53 \%$ & 549 & $25 \%$ & 260 & $27 \%$ & 70 & $6,8 \%$ \\
\hline
\end{tabular}

Tabela 2. Algumas tolerâncias dimensionais aplicáveis para aços planos conforme a ASTM A568, ${ }^{(9)}$ de acordo com o grau de resistência

\begin{tabular}{|c|c|c|c|c|}
\hline \multirow[t]{2}{*}{ Espessura (mm) } & \multirow[t]{2}{*}{ Grau de Resistência } & \multicolumn{3}{|c|}{ Afastamento Superior e Inferior na Espessura por faixa de Largura (mm) } \\
\hline & & $812<L \leq 1016$ & $1016<\mathrm{L} \leq 1219$ & $1219<\mathrm{L} \leq 1624$ \\
\hline \multirow{2}{*}{$2,08<\mathrm{E} \leq 2,49$} & Baixa e Média & 0,17 & 0,17 & 0,17 \\
\hline & Alta & 0,20 & 0,20 & 0,20 \\
\hline \multirow{2}{*}{$2,49<\mathrm{E} \leq 4,57$} & Baixa e Média & 0,17 & 0,20 & 0,20 \\
\hline & Alta & 0,20 & 0,25 & 0,25 \\
\hline \multirow{2}{*}{$4,57<\mathrm{E} \leq 5,84$} & Baixa e Média & 0,20 & 0,22 & 0,25 \\
\hline & Alta & 0,22 & 0,25 & 0,25 \\
\hline
\end{tabular}

Observação: Aços com limite de escoamento acima de $340 \mathrm{MPa}$ são considerados de alta resistência; entre $310 \mathrm{MPa}$ e 340 MPa (inclusive) são considerados de média e menores que 3 IOMPa são aços de baixa resistência, segundo a ASTM. ${ }^{(9)}$ 
Também devem ser tomados cuidados quanto ao tipo de aço selecionado. Um erro muito comum é a substituição de aços utilizados em longarinas dobradas, por exemplo, o NBR 6656 LNE $38^{(10)}$ pelo aço estrutural ASTM A572 Gr. 55. ${ }^{(8)}$ Neste caso, ambos os aços possuem o mesmo limite de escoamento mínimo (380 MPa), porém a conformabilidade garantida do aço NBR 6656 é maior que a do ASTM A572, não sendo recomendável fazer essa substituição mesmo que o aço ASTM seja mais comum e tenha menor custo.

\subsection{Resistência Mecânica}

O pilar da redução de peso das estruturas está na utilização de materiais mais resistentes. Eles proporcionam peças e componentes mais finos e leves e que apresentam a capacidade de suportar a mesma carga absoluta. $O$ principal parâmetro de projeto utilizado para comparação e para definir as substituições de material é o limite de escoamento (LE). Contudo, outros parâmetros também devem ser verificados, pois possuem influência no desempenho do material que será utilizado. São exemplos: alongamento, o limite de resistência (LR), expoente de encruamento (n) e coeficiente de anisotropia ( $R$ ). No caso do alongamento é necessário explicitar o tipo do corpo-de-prova (CP) e base de medida $\left(\mathrm{L}_{0}\right)$, pois os valores de alongamento também são dependentes destes parâmetros. ${ }^{(1)}$

É bastante difundido o uso de aços microligados ao nióbio e também ao titânio e vanádio, como forma de obter uma maior resistência mecânica aliado a um baixo teor em carbono equivalente $\left(\% \mathrm{C}_{\text {eq }}\right)$, com ganhos principalmente em soldabilidade e conformabilidade. Mais recentemente, novos aços têm surgido com características ainda mais atraentes, ${ }^{(12)}$ mas com questões ainda a serem resolvidas em conjunto entre produtores, consumidores e projetistas. ${ }^{(6,13)}$

\subsection{Soldabilidade}

Em qualquer processo de soldagem é desejável um metal com boa soldabilidade. Neste caso, o conceito de aço de boa soldabilidade é aquele que não apresente susceptibilidade à formação de defeitos e/ou heterogeneidades na zona termicamente afetada pelo calor da solda (ZTA), em variadas situações ou procedimentos de soldagem.

Mudanças muito radicais na resistência mecânica dos aços implicam necessariamente em mudanças no procedimento de solda e no metal de adição utilizado. Mesmo um ligeiro ajuste no procedimento de solda, devido à mudança do metal, pode ser um fator limitante para essa substituição. Assim, aços de maior resistência mecânica tendem a apresentar soldabilidade mais limitada.

A norma API 5L, ${ }^{(14)}$ que é uma das mais rígidas em relação à soldabilidade, limita o carbono equivalente de aços de alta resistência em 0,43\%p, conforme definido pela fórmula do International Institute of Welding (IIW):

$\% \mathrm{C}_{\text {eq }}=\% \mathrm{C}+\% \mathrm{Mn} / 6+(\% \mathrm{Mo}+\% \mathrm{Cr}+\% \mathrm{~V}) / 5+(\% \mathrm{Ni}+\% \mathrm{Cu}) / 15$

Tecnol. Metal. Mater. Miner., São Paulo, v. 6, n. I, p. I-6, jul.-set. 2009
O ideal é utilizar aços cujas estruturas e composições tenham soldabilidade tão boa quanto aquela dos aços que estão sendo substituídos.

\subsection{Conformabilidade}

Dobramentoe estampagem são os principais processos que exigem do material uma ductilidade mínima para sua utilização nos processos de fabricação de componentes e estruturas de veículos. A diminuição da espessura das peças é um fator que auxilia a sua conformabilidade, especialmente em dobramento. Porém, o incremento de resistência limita a sua capacidade de deformação e aumenta o efeito mola (também conhecido com springback). ${ }^{(6)}$ Um material com maior nível de resistência normalmente apresenta ductilidade mais restrita, o que pode implicar na ocorrência de trincas como as ilustradas na Figura I.

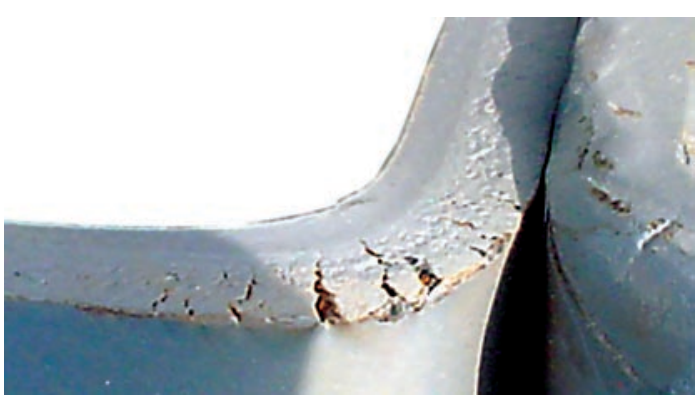

Figura I. Trincas oriundas do processo de conformação (dobramento) de um aço com alta resistência mecânica.

Peças de aço podem ser cortadas por estampo ou por laser. No caso do corte em estampo, cujo processo está mostrado esquematicamente pela Figura 2, rebarbas e rugosidades deixadas pelo processo tornam-se pontos de concentração de tensões e deformações que podem induzir trincas após a conformação, como as mostradas na Figura I. Aços com maior nível de resistência mecânica, além de apresentarem uma diferente morfologia de acabamento no corte por estampo e de aumentarem o desgaste das ferramentas de estampo, são mais sensíveis ao nível de acabamento pelo corte por estampo. Assim, devem ser feitos ajustes nos equipamentos de corte para adequar o processo ao emprego de aços de maior resistência mecânica.

É possível melhorar a conformabilidade de aços de maior resistência mecânica, durante o processo de fabricação, especialmente: i) pela alteração da morfologia das inclusões (com emprego de cálcio, titânio ou metais de terras raras), ii) pela redução do teor de enxofre e iii) pelo emprego de mecanismos de endurecimento mais adequados, por exemplo, o refino de grão. ${ }^{(16)}$ 


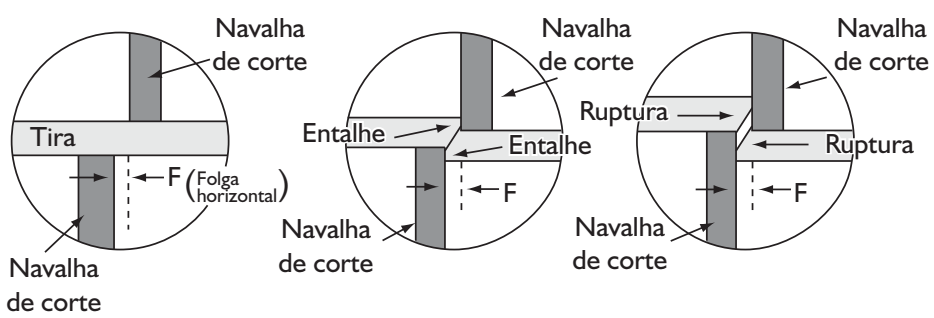

Figura 2. Corte por estampo: o tamanho relativo da zona de cisalhamento (nick) depende de uma série de características do processo, como da folga entre as ferramentas e resistência mecânica do aço cortado. ${ }^{(15)}$

\subsection{Rigidez e Empenamento}

O uso de aços de maior resistência mecânica possibilita o emprego de componentes mais finos e, portanto, mais leves. Porém, o módulo de rigidez (ou módulo de Young) dos aços é praticamente constante entre $180 \mathrm{GPa}$ a $205 \mathrm{GPa},{ }^{(17)}$ para qualquer nível de resistência mecânica. ${ }^{(16)}$ Assim, a rigidez do componente é reduzida à medida que se reduz o seu momento de inércia (ou sua espessura) e essa diminuição na rigidez pode levar a maior geração de vibrações, ruídos, desgastes e mesmo induzir fadiga.

Por outro lado, a menor espessura também torna o material mais susceptível ao empenamento em uso, que é a deformação elástica seguida de instabilidade plástica ou dobramento (flambagem distorcional) em componentes muito finos. A capacidade de um componente em empenar está diretamente associada ao momento de inércia do componente, função da razão entre a largura livre $\left(b_{f}\right)$ e a espessura $(t)$ desse componente $\left(b_{f} / t\right)$. Desta forma, para obter uma redução de peso efetiva, deve-se diminuir a espessura do componente:

- sem maiores ajustes, quando a rigidez do componente é pouco pronunciada; ou

- compensando a perda de rigidez pela alteração da geometria do componente/estrutura.

\subsection{Custo}

Existe uma série de vantagens técnicas no uso de aços de alta resistência mecânica para a produção de estruturas e componentes veiculares. Entretanto, $\bigcirc$ custo inicial do projeto não será necessariamente diminuído com o emprego desses aços. A Tabela 3 exemplifica o (sobre) custo relativo existente entre cinco aços estruturais diferentes conforme obtido da bibliografia. ${ }^{(17)}$ Salienta-se que 0 custo real dos aços depende de vários fatores, ${ }^{(16)}$ sendo que esta tabela serve apenas como referência.

Neste caso, a substituição do aço ASTM A36(7) pelo ASTM A572 Gr. 50 ${ }^{(8)}$ implica em $39 \%$ de aumento de resistência e em $9,4 \%$ de aumento de custo. ${ }^{(17)}$ Mas esse tipo de substituição tem o potencial de diminuir efetivamente o custo inicial da peça ou componente, pois os ganhos na diminuição de peso, pelo aumento da resistência, são maiores do que $\circ$ incremento no custo desse material, o que aumenta a competitividade do componente.

A utilização de aços com maior resistência à corrosão pode não levar a ganhos imediatos de custo (Tabela 3 para o caso do aço ASTM A242). Contudo, em longo prazo, os ganhos em redução do consumo de combustível, aumento da capacidade (carga paga) e ampliação da longevidade da estrutura podem compensar, em muito, um custo inicial mais elevado.

\subsection{Propostas de Substituição}

Ao se considerar todos os itens analisados, propuseram-se substituições de aços para clientes da Usina de Cubatão que atuam no setor de implementos rodoviários. Nestas propostas de substituição, visaram-se:

- redução imediata do custo total da estrutura;

- aumento da resistência mecânica e redução do peso total da estrutura;

- manutenção das condições de fabricação ou mínima influência sobre estas;

- menor impacto sobre as tolerâncias dimensionais, de forma e na rigidez da estrutura final; e

- melhora do desempenho e a qualidade geral dos produtos do cliente.

Estes objetivos foram atingidos por:

Tabela 3. Relações entre a resistência mecânica e custo de alguns aços estruturais ${ }^{(17)}$

\begin{tabular}{|c|c|c|c|c|c|}
\hline \multirow[t]{2}{*}{ Especificação } & \multirow[t]{2}{*}{ Tipo de Aço } & \multicolumn{2}{|c|}{$\begin{array}{c}\text { Limite de } \\
\text { escoamento }\end{array}$} & \multicolumn{2}{|c|}{ (\%) Aumento em relação ao A36 } \\
\hline & & $\mathrm{MPa}$ & ksi & Na resistência & No custo ${ }^{a}$ \\
\hline ASTM A36 & Carbono comum & 248 & 36 & - & - \\
\hline ASTM A572 Grau 50 & Microligado ao nióbio e vanádio & 345 & 50 & 38,9 & 9,4 \\
\hline ASTM A440 & $\mathrm{Mn}-\mathrm{Cu}$ & “ & “ & “ & 13,4 \\
\hline ASTM A44I & $\mathrm{Mn}-\mathrm{V}-\mathrm{Cu}$ & “ & “ & “ & “ \\
\hline ASTM A242 & Patinável & “ & “ & “ & 38,3 \\
\hline
\end{tabular}

${ }^{\text {a} B a s e a d o ~ n o ~ v a l o r ~ d e ~ p a r i d a d e ~ d o ~ d o ́ l a r ~ d e ~} 1970$. 
- substituição de aços de menor nível de resistência, por aços de nível de resistência imediatamente superior,

- diminuição da espessura, visando adotar a espessura padrão imediatamente inferior à inicialmente adotada,

- demonstração para os clientes da redução de custo proveniente e posteriores vantagens; e

- assessoria direta do corpo técnico da Usiminas junto aos seus clientes.

\section{RESULTADOS E DISCUSSÃO}

Foram feitas várias análises em conjunto com clientes, dentre as quais, foi escolhido um caso para ilustrar a filosofia deste trabalho. A Figura 3 ilustra uma carreta utilizada para o recolhimento de cana-de-açúcar, cuja estrutura consiste por grupos de componentes tubulares, de seção reta quadrada e com espessuras de 6,30 mm e 12,70 mm, que eram constituídos do aço ASTM A36. (7) Neste caso, o principal objetivo é tornar a estrutura mais leve. A estrutura da carreta é dividida, conforme definido pelo fabricante, em quatro conjuntos: caçamba, chassi, elevador e rodagem.

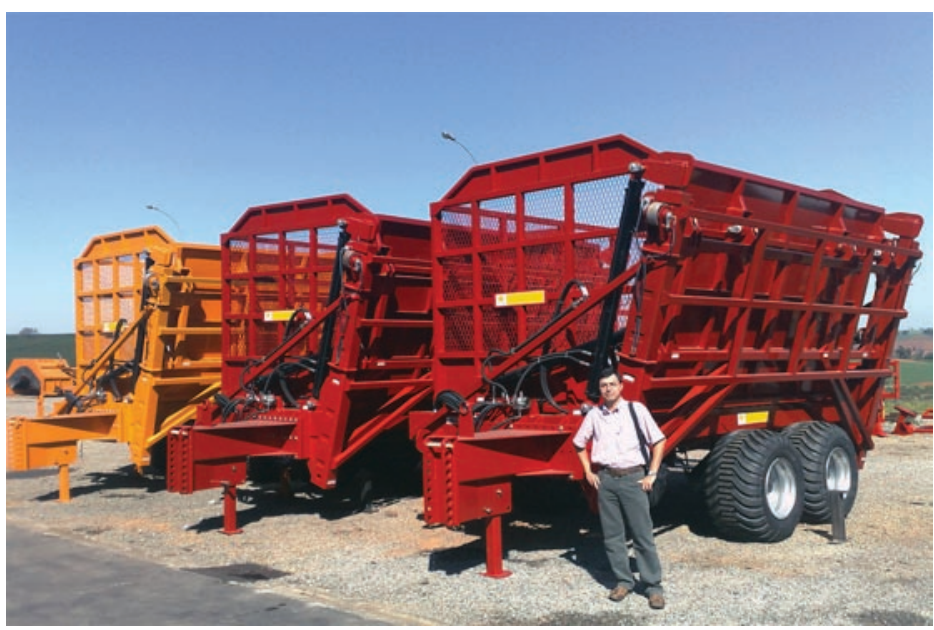

Figura 3. Carretas para o transporte de cana-de-açúcar durante a colheita.
Foram analisados três tipos de aços em substituição ao $A 36,{ }^{(7)}$ tomando, em consenso com o produtor, o limite de escoamento como parâmetro básico de desempenho. A Tabela 4 ilustra os valores dos limites de escoamento dos aços analisados como possíveis substitutos ao $A 36^{(7)}$ e o e aumento de resistência associado a cada substituição.

Neste caso, o potencial de redução de peso deve levar em consideração a possibilidade de utilizar a "espessura padrão", citada no item 2.I, o que leva aos valores mostrados na Tabela 5.

Considerando os critérios do item 2.7, sugeriu-se a substituição do ASTM A36 (7) pelo ASTM A572 Gr. 50. ${ }^{(8)}$ O resultado final de redução de peso para esta substituição está mostrada na Tabela 6. O fabricante realizou ajustes para compensar a perda de rigidez da estrutura, alterando o posicionamento de alguns componentes, sem alterar a quantidade total destes na estrutura como um todo.

Há um ganho em peso final de $25 \%$ que ainda serão acompanhados por uma redução em custo inicial para a fabricação do produto, conforme apontado pelos dados da Tabela $3 .^{(17)} \mathrm{A}$ presente substituição também permite um incremento na vida útil do componente, já que houve

Tabela 4. Comparação entre os limites de escoamento (LE) mínimo de garantia de algumas normas de aços estruturais aplicáveis para a carreta

\begin{tabular}{|c|c|c|}
\hline Tipo de aço & $\begin{array}{c}\text { LE mínimo } \\
\text { garantido } \\
(\mathrm{MPa})\end{array}$ & $\begin{array}{c}\text { Incremento } \\
\text { na resistência } \\
(\%)\end{array}$ \\
\hline ASTM A36(7) & 250 & $0 \%$ \\
\hline ASTM A572 Gr.50(8) & 345 & $38 \%$ \\
\hline NBR 6656 LNE 380(10) & 380 & $52 \%$ \\
\hline NBR 6656 LNE 600(10) & 600 & $140 \%$ \\
\hline
\end{tabular}

Tabela 5. Ajustes nas espessuras de alguns aços analisados para aplicar na carreta de cana-de-açúcar em face das "espessuras padrão"

\begin{tabular}{|c|c|c|c|c|c|c|}
\hline \multirow[t]{2}{*}{ Tipo de aço } & \multicolumn{6}{|c|}{ Espessuras (mm) } \\
\hline & Inicial & Teórica & Real & Inicial & Teórica & Real \\
\hline ASTM A36(7) & \multirow{4}{*}{6,30} & 6,30 & 6,30 & \multirow{4}{*}{12,70} & 12,70 & 12,70 \\
\hline ASTM A572 Gr.50(8) & & 4,57 & 4,75 & & 9,20 & 9,50 \\
\hline NBR 6656 LNE 380(10) & & 4,14 & 4,25 & & 8,36 & 9,50 \\
\hline NBR 6656 LNE $600^{(10)}$ & & 2,63 & 2,65 & & 5,29 & 6,30 \\
\hline
\end{tabular}

Tabela 6. Possíveis substituições de material e respectiva redução de peso nas quatro partes constituintes da carreta de cana-de-açúcar

\begin{tabular}{|c|c|c|c|c|c|}
\hline Partes & Participação & Espessura (mm) & ASTM A36(7) (kg) & $\begin{array}{l}\text { ASTM A572 } \\
\mathrm{Gr.50}^{(8)}(\mathrm{kg})\end{array}$ & Redução de peso \\
\hline Caçamba & $28 \%$ & 6,30 & 2.020 & 1.523 & \multirow{4}{*}{$25 \%$} \\
\hline Chassi & $38 \%$ & 6,30 & 2.750 & 2.073 & \\
\hline Elevador & $19 \%$ & 12,70 & 1.050 & 785,4 & \\
\hline Rodagem & $17 \%$ & 12,70 & 996 & 745 & \\
\hline & Total & & 6.816 & 5.127 & 1,7 ton \\
\hline
\end{tabular}


um ganho final em resistência mecânica e, conseqüentemente, em resistência à fadiga. Com isso o desempenho do equipamento foi incrementado, aumentando sua aceitação no mercado.

\section{CONCLUSÕES}

O trabalho foi desenvolvido em resposta à contínua necessidade de redução de custo e ganho em desempenho de produtos, especialmente no competitivo segmento de implementos rodoviários. As análises feitas indicam que, para realizar substituições de material para a redução de peso, não basta apenas considerar o grau de resistência projetada e garantida do aço ${ }^{(16)} \mathrm{e}$ sim outros fatores, $(2,3,6,12,13)$ especialmente os descritos neste trabalho. Nesta linha foi possível realizar vários casos práticos de substituição de aço, com vantagens para o produtor da matériaprima, da estrutura e do usuário final, como o ilustrado neste trabalho.

\section{REFERÊNCIAS}

I AISI-American Iron and Steel Institute. The ultralight steel auto body (ULSAB). Disponível em: <http://www.autosteel. org/AM/Template.cfm?Section=ULSABI >. Acesso em: 31 mar. 2008.

2 IBS - Instituto Brasileiro de Siderurgia. A participação do aço nos automóveis: relatório da análise feita pelo Instituto de Pesquisas Tecnológicas (IPT). Disponível em: <http://www.ibs.org.br/index.asp>. Acesso em: I mar. 2008.

3 SSAB Swedish Steel. New supersteels help the automotive industry meet environmental targets. Disponível em: <http:// www.ssabtunnplat.com/templates/PressPage.aspx?id=3568>. Acesso em: 28 mar. 2008.

4 EUROPEAN COMMITTEE FOR STANDARDIZATION/DEUTSCHES INSTITUT FÜR NORMUNG. DIN EN I0268: Cold-rolled flat products made of high yield strength micro-alloyed steels for cold forming: technical delivery conditions (English version of DIN EN 10268). Brussels, 1999.

5 RABBI, M.S. Fadiga dos metais. In: MORAIS, W.A.; MAGNABOSCO, A.S.; MENEZES NETTO, E.B. Metalurgia física e mecânica. São Paulo: ABM, 2007. V. 2, p. 40I-24.

6 SILVA, A.T. Análise das implicações do uso dos novos aços de alta resistência mecânica na industria automobilística. In: CONGRESSO ANUAL DA ABM, 59., 2004, São Paulo. Anais... São Paulo: ABM, 2004. p. 335-42, I CD-Rom.

7 ASTM INTERNATIONAL. ASTM A36/A36M: standard specification for carbon structural steel. West Conshohocken, 2007.

8 ASTM INTERNATIONAL. ASTM A572/A572M-07: standard specification for high-strength low-alloy columbiumvanadium structural steel. West Conshohocken, 2007.

9 ASTM INTERNATIONAL. ASTM A568/A568M-07a: Standard Specification for Steel, Sheet, Carbon, Structural, and High-Strength, Low-Alloy, Hot-Rolled and Cold-Rolled, General Requirements for. West Conshohocken, 2007.

10 ABNT - ASSOCIAÇÃO BRASILEIRA DE NORMAS TÉCNICAS. NBR 6656: Bobinas e chapas laminadas a quente de aço acalmado com características especiais de propriedades mecânicas, conformabilidade e soldabilidade: Especificação. Rio de Janeiro, 2008.

II INTERNATIONAL ORGANIZATION FOR STANDARDIZATION. ISO 2566/I: Steels - Conversion of elongation values- Part I: Carbon and low alloy steels. 2. ed. Geneva, 1984.

12 MILITITSKY, M. Advanced steel products for Lightweighting at Daimlerchrysler. In: HASHIMOTO, S.; JANSTO, S.; MOHRBACHER, H; SICILIANO, F. In: INTERNATIONAL SYMPOSIUM ON NIOBIUM MICROALLOYED SHEET STEEL FOR AUTOMOTIVE APPLICATIONS, 2005, Araxá, MG. Proceedings... Warrendale: The Minerals, Metals \& Materials Society, 2006. p. 53-63, I CD-Rom.

13 KIESE, J. New lightweight steels for automotive applications: potential and risk. In: HASHIMOTO, S.; JANSTO, S.; MOHRBACHER, H; SICILIANO, F. In: INTERNATIONAL SYMPOSIUM ON NIOBIUM MICROALLOYED SHEET STEEL FOR AUTOMOTIVE APPLICATIONS, 2005, Araxá, MG. Proceedings... Warrendale: The Minerals, Metals \& Materials Society, 2006. p. 89-97, I CD-Rom.

I4 API - AMERICAN PETROLEUM INSTITUTE. API 5L: specification for line pipe. 43. ed. New York, 2004.

15 THEIS, H. E. Handbook of metalforming process. New York: Marcel Dekker, 1999.

16 MORAIS, W.A.; BORGES, H.C.; PEREZ, F.A. Modelamento e quantificação da relação propriedades x microestrutura nos aços estruturais laminados a quente da Cosipa. In: CONGRESSO ANUAL DA ABM, 62, 2007, Vitória, ES. Anais... São Paulo: ABM, 2007. p. 3285-96, I CD-Rom.

17 BAUCCIO, M. ASM metals reference book. 3. ed. Materials Park: American Society for Metals, I993. p. I I3.

Recebido em: 29/03/2009

Aprovado em: 12/08/2009 